\title{
Heat Transfer Modeling of the Ground Heat Exchangers for the Ground-Coupled Heat Pump Systems
}

\author{
Yi Man, Ping Cui and Zhaohong Fang \\ Key Laboratory of Renewable Energy Utilization Technologies in Buildings, \\ Ministry of Education, China; Shandong Jianzhu University, Jinan, Shandong, \\ China
}

\section{Introduction}

Ground-coupled heat pump (GCHP) systems have been gaining increasing popularity for space air conditioning in buildings due to their reduced energy and maintenance costs. The efficiency of GCHP systems is inherently higher than that of the traditional options because it utilizes the ground which maintains a relatively stable temperature all the year round as a heat source/sink. Compared with traditional air-conditioning systems, the GCHP system features its ground heat exchanger (GHE), whether it is horizontally installed in trenches or as U-tubes in vertical boreholes. The advantages of vertical GHEs are that they require smaller plots of land areas, and can yield the most efficient GCHP system performance. The vertical GHEs are usually constructed by inserting one or two high-density polyethylene Utubes in vertical boreholes, which are referred to as single U-tube or double U-tube GHEs, respectively. The boreholes should be grouted to provide better thermal conductance and prevent groundwater from possible contamination. Borehole depths usually range from 40 to 200 meters with diameter of 100 to 150 millimeters. The schematic diagram of a borehole with U-tubes in vertical GHEs is illustrated in Fig. 1.

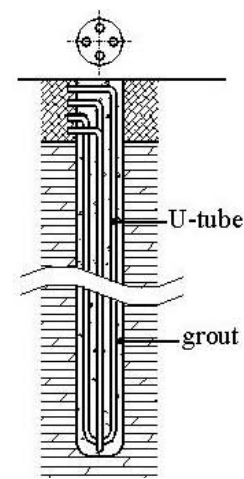

a) double U-tube

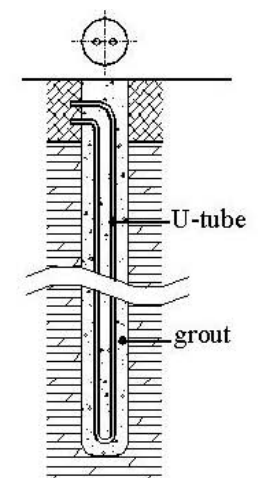

b) single U-tube

Fig. 1. Schematic diagram of boreholes in the vertical GHE exchanger 
However, the commercial growth of the GCHP systems has been hindered by its higher capital cost, of which a significant portion is attributed to the GHE. Besides the structural and geometrical configuration of the exchanger a lot of factors influence the exchanger performance, such as the ground temperature distribution, soil moisture content and its thermal properties, groundwater movement and possible freezing and thawing in soil. Thus, heat transfer between a GHE and its surrounding soil/rock is difficult to model for the purpose of sizing the GHE or simulation of the GCHP systems. In order to assess the thermal behaviour and to optimise the technical as well as economical aspects of GCHP systems, it is crucial to work out appropriate and validated heat transfer models of the GHE.

To determine the heat transfer in the GHEs with adequate accuracy is a crucial task, and has great impact on sizing and simulating GHE. The design goal is to control the temperature rise of the ground and the circulating fluid within acceptable limits over the lifetime of the system. A fundamental task for application of the GCHP technology is to grasp the heat transfer process of a single borehole in the GHE. Heat transfer in a field with multiple boreholes may be analyzed on this basis with the superposition principle.

There are roughly two categories of approaches in dealing with the thermal analysis and design of the GHEs. Empirical or semi-empirical formulations are recommended in textbooks and monographs for GHE design purposes (Bose et al., 1985; Kavanaugh, 1997). These approaches are relatively simple, and may be manipulated easily by design engineers. However, they do not reveal in detail the impacts of complicated factors on the GHE performance. The second kind of approaches involves numerical simulation of the heat transfer in the GHEs (Mei \& Baxter, 1986; Yavuzturk \& Spitler, 1999). While having provided important understandings on GHE heat transfer, these studies of numerical simulation have not yet been suitable to design and/or energy analysis of full scale engineering projects because it takes too substantial computing time.

Theoretical study on the GHE with an analytical approach is presented by some Swedish and American scholars (Eskilson, 1987; Spitler, 2005). Involving a time span of several years, the heat transfer process in the ground around the vertical boreholes is rather complicated, and should be treated, on the whole, as a transient one. Because of all the complications of this problem and its long time scale, the heat transfer process may usually be analyzed in two separated regions. One is the solid soil/rock outside the borehole, where the heat conduction must be treated as a transient process. With the knowledge of the temperature response in the ground, the temperature on the borehole wall can then be determined for any instant on specified operational conditions. Another sector often segregated for analysis is the region inside the borehole, including the grout, the U-tube pipes and the circulating fluid inside the pipes. The main objective of this analysis is to determine the inlet and outlet temperatures of the circulating fluid according to the borehole wall temperature, the thermal resistance inside the borehole and the heat rate of the GHE.

In this approach for GHE analysis a single borehole is investigated in detail experiencing a step heating/cooling. Then, the principle of superimposition is used to deal with the more complicated situation of GHEs with multiple boreholes as well as the variable load. It is more adequate and accurate than the empirical approaches and yet much more convenient for computations than the numerical simulations. In this regard, better understanding of every thermal resistances of the GHE is crucial, and their analytical solutions are especially 
preferred to facilitate the computation. Following such an approach, some important analytical solutions have been derived by our research group (Diao \& Fang, 2006) for heat transfer processes both inside and outside the boreholes, which can be easily incorporated into computer programs for thermal analysis and sizing of the GHEs while providing better insight into influences of various factors on the GHE performance.

In practice, the boreholes of GHEs may penetrate several geologic strata. It is desirable to account for the groundwater flow in the heat transfer model to avoid over-sizing of the GHE. Taking the groundwater advection into account, the combined heat transfer of conduction and advection in the GHE has been solved by an analytical approach, and explicit expressions of the temperature response has been derived (Diao et al., 2004; Nelson et al., 2011).

Recently, a novel configuration of GHE with a spiral coil has been proposed and applied in practical projects due to its distinct thermal and economical advantages especially combined with the foundation piles of buildings. For better simulating the heat transfer of buried spiral coils, our research group have proposed two new kinds of models and resolved their analytical solutions (Cui et al., 2011; Man et al., 2011).

This chapter analyzes in detail every links of the heat transfer process in borehole heat exchangers, including the influence of the groundwater movement. Adequate analytical solutions are suggested for modeling of the GHEs. The superposition procedures for multiple boreholes and variable loads are also discussed to provide an integrated solution of the thermal analysis of the GHEs. This approach can be easily incorporated into computer programs for thermal analysis and sizing of the GHEs while providing better insight into influences of various factors on the GHE performance.

\section{Heat transfer inside boreholes}

\subsection{Overview}

The main objective to analyze the heat transfer inside boreholes is to determine the entering and leaving temperatures of the circulating fluid in the GHE according to the borehole wall temperature and its heat flow. Compared with the infinite ground outside it, both the dimensional scale and thermal mass of the borehole are much smaller. Moreover, the temperature variation inside the borehole is usually slow and minor. Thus, it is a common practice that the heat transfer in this region is approximated as a steady-state process.

It is obvious that the double U-tube configuration provides more heat transfer area between the circulating fluid and the ground than the single U-tube GHE does, and will reduce thermal resistance inside the borehole. On the other hand, however, it might require more pipes and consume more pumping power on operation for a certain project. Thus, analysis on performance and costs of different configurations of the GHEs has been a task for scholars and engineers to study.

A few models of varying complexity have been established to describe the heat transfer inside the GHE boreholes. Models for practical engineering designs are often oversimplified in dealing with the complicated geometry inside the boreholes. One-dimensional (1-D) model (Bose et al., 1985) has been recommended for engineering design, conceiving the U- 
tube pipes as a single "equivalent" pipe. By a different approach Hellstrom (1991) has derived two-dimensional (2-D) analytical solutions of the borehole thermal resistances in the cross-section perpendicular to the borehole. On assumptions of identical temperatures and heat fluxes of all the pipes in it, the borehole resistance was worked out. Exchanging heat with the surrounding ground, however, the fluid circulating through different legs of the Utubes is of varying temperatures. As a result, thermal interference, or thermal "shortcircuiting", among U-tube legs is inevitable, which degrades the effective heat transfer in the GHEs. With the assumption of identical temperature of all the pipes, it is impossible for all the models mentioned above to reveal impact of this thermal interference on GHE performances.

On the other hand, Mei \& Baxter (1986) considered the 2-D model of the radial and longitudinal heat transfer, which was solved with a finite difference scheme. Yavuzturk et al. (1999) employed the 2-D finite element method to analyze the heat conduction in the plane perpendicular to the borehole for short time step responses. Requiring numerical solutions, these models are of limited practical value for use by designers although they may result in more exact solutions for research and parametric analysis of GHEs.

Taking the fluid axial convective heat transfer and thermal "short-circuiting" among U-tube legs into account, a quasi three-dimensional (3-D) model for boreholes in GHEs has been established as an extension of the work of Eskilson (1987) and Hellstrom (1991) to reveal the thermal interference between the U-tube legs. Analytical solutions of the fluid temperature profiles along the borehole depth have been obtained (Zeng et al., 2003). This model takes into account more factors than previous models ever did before, including the geometrical parameters (borehole and pipe sizes and pipe disposal in the borehole) and physical parameters (thermal conductivity of the materials, flow rate and fluid properties) as well as the flow circuit configuration. Its solutions have provided a reliable tool for GHE sizing and performance simulation and a solid basis for technical and economic assessment of different borehole configurations.

\subsection{Quasi 3-D model on heat transfer inside the borehole}

This section focuses on the model of heat transfer inside the borehole taking into account the 2$\mathrm{D}$ heat conduction in the transverse cross-section as well as the convective heat transfer in the axial direction by the fluid inside the U-tubes, which is referred to as the quasi 3-D model. To keep the problem analytically manageable some simplifications are assumed. They are:

1. The heat capacity of the materials inside the borehole is neglected.

2. The heat conduction in the axial direction is negligible, and only the conductive heat flow among the borehole wall and the pipes in the transverse cross-section is counted.

3. The borehole wall temperature, $T_{\mathrm{b}}$, is constant along its depth, but may vary with time.

4. The ground outside the borehole and grout in it are homogeneous, and all the thermal properties involved are independent of temperature.

Number the pipes in the borehole clockwise as shown in Fig. 2. If the temperature on the borehole wall is taken as the reference of the temperature excess, the fluid temperature excess in the pipes may be expressed as the sum of four separate temperature excesses caused by the heat fluxes per unit length, $q_{1}, q_{2}, q_{3}$ and $q_{4}$ from the four legs of the U-tubes. Thus, the following expressions may be obtained 


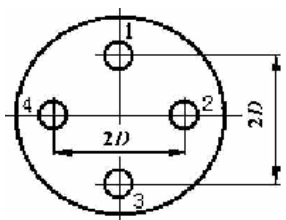

Fig. 2. Cross-section of a borehole with double U-tube

$$
\left.\begin{array}{l}
T_{f 1}-T_{b}=R_{11} q_{1}+R_{12} q_{2}+R_{13} q_{3}+R_{14} q_{4} \\
T_{f 2}-T_{b}=R_{21} q_{1}+R_{22} q_{2}+R_{23} q_{3}+R_{24} q_{4} \\
T_{f 3}-T_{b}=R_{31} q_{1}+R_{32} q_{2}+R_{33} q_{3}+R_{34} q_{4} \\
T_{f 4}-T_{b}=R_{41} q_{1}+R_{42} q_{2}+R_{43} q_{3}+R_{44} q_{4}
\end{array}\right\}
$$

where $R_{\mathrm{ii}}(\mathrm{i}=1,2,3,4)$ is the thermal resistance between the circulating fluid in a certain Utube leg and the borehole wall, and $R_{\mathrm{ij}}(\mathrm{i}, \mathrm{j}=1,2,3,4)$ the resistance between two individual pipes. It is most likely in engineering practice the U-tube legs are disposed in the borehole symmetrically as shown in Fig. 2 . In this case one gets $R_{\mathrm{ij}}=R_{\mathrm{ji}}, R_{\mathrm{ii}}=R_{\mathrm{jj}}(\mathrm{i}, \mathrm{j}=1,2,3,4)$ and $R_{14}=R_{12}$ and so on. Hellstrom (1991) analyzed the steady-state conduction problem in the borehole cross-section in detail with the line-source and multiple approximations. The linesource assumption has resulted in the following solution.

$$
\begin{aligned}
& R_{11}=\frac{1}{2 \pi k_{b}}\left[\ln \left(\frac{r_{b}}{r_{P}}\right)-\frac{k_{b}-k}{k_{b}+k} \ln \left(\frac{r_{b}^{2}-D^{2}}{r_{b}^{2}}\right)\right]+R_{P} \\
& R_{12}=\frac{1}{2 \pi k_{b}}\left[\ln \left(\frac{r_{b}}{\sqrt{2} D}\right)-\frac{k_{b}-k}{2\left(k_{b}+k\right)} \ln \left(\frac{r_{b}^{4}+D^{4}}{r_{b}^{4}}\right)\right] \\
& R_{13}=\frac{1}{2 \pi k_{b}}\left[\ln \left(\frac{r_{b}}{2 D}\right)-\frac{k_{b}-k}{k_{b}+k} \ln \left(\frac{r_{b}^{2}+D^{2}}{r_{b}^{2}}\right)\right]
\end{aligned}
$$

where $k$ denotes the conductivity of soil/rock around the borehole, while $k_{\mathrm{b}}$ the heat conductivity of the grouting material, and $R_{\mathrm{p}}$ the heat transfer resistance from the fluid inside the U-tubes to the pipe outside surface.

A linear transformation of equation (2) leads to:

$$
\left.\begin{array}{l}
q_{1}=\frac{T_{f 1}-T_{b}}{R_{1}^{\Delta}}+\frac{T_{f 1}-T_{f 2}}{R_{12}^{\Delta}}+\frac{T_{f 1}-T_{f 3}}{R_{13}^{\Delta}}+\frac{T_{f 1}-T_{f 4}}{R_{12}^{\Delta}} \\
q_{2}=\frac{T_{f 2}-T_{f 1}}{R_{12}^{\Delta}}+\frac{T_{f 2}-T_{b}}{R_{1}^{\Delta}}+\frac{T_{f 2}-T_{f 3}}{R_{12}^{\Delta}}+\frac{T_{f 2}-T_{f 4}}{R_{13}^{\Delta}} \\
q_{3}=\frac{T_{f 31}-T_{f 1}}{R_{13}^{\Delta}}+\frac{T_{f 3}-T_{f 2}}{R_{12}^{\Delta}}+\frac{T_{f 1}-T_{b}}{R_{1}^{\Delta}}+\frac{T_{f 3}-T_{f 4}}{R_{12}^{\Delta}} \\
q_{4}=\frac{T_{f 4}-T_{f 1}}{R_{12}^{\Delta}}+\frac{T_{f 4}-T_{f 2}}{R_{13}^{\Delta}}+\frac{T_{f 4}-T_{f 3}}{R_{12}^{\Delta}}+\frac{T_{f 4}-T_{b}}{R_{1}^{\Delta}}
\end{array}\right\}
$$


where

$$
\begin{gathered}
R_{1}^{\Delta}=R_{11}+R_{13}+2 R_{12}, R_{12}^{\Delta}=\frac{R_{11}^{2}+R_{13}^{2}+2 R_{11} R_{13}-4 R_{12}^{2}}{R_{12}}, \\
R_{13}^{\Delta}=\frac{\left(R_{11}-R_{13}\right)\left(R_{11}^{2}+R_{13}^{2}+2 R_{11} R_{13}-4 R_{12}^{2}\right)}{R_{13}^{2}+R_{11} R_{13}-2 R_{12}^{2}} .
\end{gathered}
$$

In this model the convective heat flow along the fluid channel is balanced by the conductive heat flows among the fluid channels and borehole wall. According to equation (3) the heat equilibrium of the fluid in individual pipes can be formulated as:

$$
\left.\begin{array}{l} 
\pm M c \frac{d T_{f 1}(z)}{d z}=\frac{T_{f 1}(z)-T_{b}}{R_{1}^{\Delta}}+\frac{T_{f 1}(z)-T_{f 2}(z)}{R_{12}^{\Delta}}+\frac{T_{f 1}(z)-T_{f 3}(z)}{R_{13}^{\Delta}}+\frac{T_{f 1}(z)-T_{f 4}(z)}{R_{12}^{\Delta}} \\
\pm M c \frac{d T_{f 2}(z)}{d z}=\frac{T_{f 2}(z)-T_{f 1}(z)}{R_{12}^{\Delta}}+\frac{T_{f 2}(z)-T_{b}}{R_{1}^{\Delta}}+\frac{T_{f 2}(z)-T_{f 3}(z)}{R_{12}^{\Delta}}+\frac{T_{f 2}(z)-T_{f 4}(z)}{R_{13}^{\Delta}} \\
\pm M c \frac{d T_{f 3}(z)}{d z}=\frac{T_{f 3}(z)-T_{f 1}(z)}{R_{13}^{\Delta}}+\frac{T_{f 3}(z)-T_{f 2}(z)}{R_{12}^{\Delta}}+\frac{T_{f 3}(z)-T_{b}}{R_{1}^{\Delta}}+\frac{T_{f 3}(z)-T_{f 4}(z)}{R_{12}^{\Delta}} \\
\pm M c \frac{d T_{f 4}(z)}{d z}=\frac{T_{f 4}(z)-T_{f 1}(z)}{R_{12}^{\Delta}}+\frac{T_{f 4}(z)-T_{f 2}(z)}{R_{13}^{\Delta}}+\frac{T_{f 4}(z)-T_{f 3}(z)}{R_{12}^{\Delta}}+\frac{T_{f 4}(z)-T_{b}}{R_{1}^{\Delta}}
\end{array}\right\}
$$

Here the signal \pm on the left side of the equations depends on the condition whether the fluid flows in the same direction as the $z$-coordinate, which is designated to be downwards. When the fluid moves downwards, the signal is positive, and vice versa. Combined with certain connecting conditions from the flow circuit arrangement, the energy equilibrium equation can be solved by means of Laplace transformation. Then, the temperature distribution of circulating fluid along the channels can be analytically worked out, and the thermal resistance inside the borehole can be determined more adequately.

\subsection{Fluid temperature profiles along the depth and borehole resistance}

The fluid temperature profiles in the flow channels and, then, the borehole resistance are affected by borehole configuration. As mentioned above, there are single and double U-tube boreholes. The latter can be arranged in series or parallel flow circuits, and each of them includes a few connecting patterns. For the two U-tubes in the borehole connected in parallel circuit, different combinations of circuit arrangement come down to two options that make difference to its heat transfer. They may be represented by notations of $(1-3,2-4)$ and (1-2, 3-4). Here 1-3 denotes that the fluid flows through pipes 1 and 3 as indicated in Fig. 2 , and also through pipes 2 and 4 in parallel. When the fluid circulates through the four legs of the U-tubes in a series circuit, there are quite a few possible layouts. Only three of them, however, bear different impact on the performance of GHE on the assumption of symmetrical disposal of the pipes. The three representative layouts in series are marked as 1-3-2-4, 1-2-3-4 and 1-2-4-3, where the sequence indicates flow succession of the pipes as shown in Fig. 2. 
All these options have been analyzed separately. Analytical expressions of the fluid temperature profiles along the channel have been obtained for all these option. The dimensionless solution of the temperature profile along the borehole depth takes the following form for the single U-tube and the double U-tube in parallel configurations.

$$
\left.\begin{array}{l}
\Theta_{1}(Z)=\cosh (\beta Z)-\frac{\sinh (\beta Z)}{\sqrt{1-P^{2}}}\left[1-P \frac{\cosh (\beta)-\sqrt{\frac{1-P}{1+P}} \sinh (\beta Z)}{\cosh (\beta)+\sqrt{\frac{1-P}{1+P}} \sinh (\beta)}\right] \\
\Theta_{2}(Z)=\frac{\cosh (\beta)-\sqrt{\frac{1-P}{1+P}} \sinh (\beta)}{\cosh (\beta)+\sqrt{\frac{1-P}{1+P}} \sinh (\beta)} \cosh (\beta Z)+\frac{\sinh (\beta Z)}{\sqrt{1-P^{2}}}\left[\frac{\cosh (\beta)-\sqrt{\frac{1-P}{1+P}} \sinh (\beta)}{\cosh (\beta)+\sqrt{\frac{1-P}{1+P}} \sinh (\beta)}-P\right]
\end{array}\right\}
$$

More intricate but less-frequently-used solutions for the double U-tube in series can be found elsewhere (Zeng et al., 2003) together with the definitions of the dimensionless parameters in above equations. Typical temperature profiles along the single and double Utubes in different flow patterns are plotted in Fig. 3.

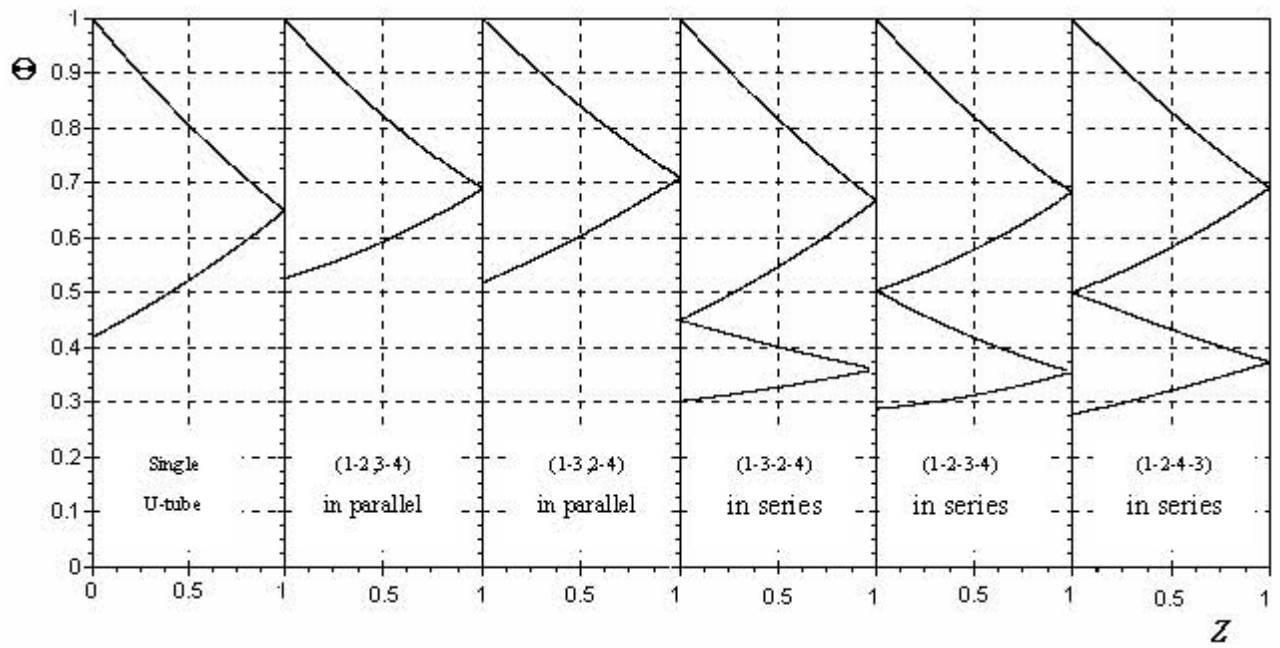

Fig. 3. Temperature profiles along the borehole depth with different U-tube configurations

The effective borehole thermal resistance defines the proportional relationship between the heat flow rate transferred by the borehole and the temperature difference between the circulating fluid and the borehole wall, that is:

$$
R_{b}=\frac{T_{f}-T_{b}}{q_{l}}
$$


where $T_{f}=\left(T_{f}^{\prime}+T_{f}^{\prime \prime}\right) / 2$ denotes the arithmetic mean of the inlet and outlet fluid temperatures. In view of heat balance for the single U-tube and double U-tube in series one also has:

$$
q_{l} H=M c\left(T_{f}^{\prime}-T_{f}^{\prime \prime}\right)
$$

As a result the borehole resistance can be determined according to the analytical solutions of fluid temperature profile in the borehole presented in previous discussion.

Combined with the definition of dimensionless temperature, equations (6) and (7) result in the following expression of borehole resistance for boreholes with the single U-tube and double U-tube in series.

$$
R_{b}=\frac{H}{2 M c} \cdot \frac{1+\Theta_{f}^{\prime \prime}}{1-\Theta_{f}^{\prime \prime}}
$$

In the case of double U-tubes in parallel the fluid mass rate in the borehole is doubled, i.e. $2 M$, thus the borehole thermal resistance can be expressed as:

$$
R_{b}=\frac{H}{4 M c} \cdot \frac{1+\Theta_{f}^{\prime \prime}}{1-\Theta_{f}^{\prime \prime}}
$$

The borehole thermal resistance defined above takes into accounts both the geometrical parameters (borehole and pipe sizes and pipe disposal in the borehole) and physical parameters (thermal conductivity of materials, flow rate and fluid properties). Therefore the concept of effective thermal resistance facilitates heat transfer analysis. Analyses have shown that the single U-tube boreholes yield considerably higher borehole resistance than the double U-tube boreholes do while the other conditions are identical. Practical choice of the U-tube configuration should be taken in accordance with economic consideration in practical engineering.

\section{Heat conduction outside boreholes}

\subsection{Overview}

There have been some classical models for GHE thermal analysis based on analytical 1-D solutions. A most widely used 1-D model for this purpose is Kelven's line source model (Carslaw \& Jeager, 1947). In this model the borehole is replaced by a line heat source with its radial dimension neglected. Another best known 1-D model, referred as the cylindrical heat source model (Carslaw \& Jeager, 1947; Ingersoll \& Zobel, 1954) is an alternative approach to sizing GHEs. Although the radial dimension of the borehole is taken into consideration in this model, the heat capacity of the cylinder is ignored, so the geometrical domain of the model can be regarded as an infinite medium with a cylindrical cavity in it. We refer it to as the "hollow" cylindrical heat source model to distinguish it from the "solid" cylindrical heat source model we are to propose below. Significant simplifications are made in these two classical models, which result in substantial deviations of the temperature response from the actual situation especially in the initial period of the heating pulse. Therefore, neither of these two models is suitable for short time-step analysis of GHEs. 
A progress in the short time-step simulations of the GHEs is the short time-step response factor model developed by Yavuzturk \& Spitler (1999) based on numerical solution by taking the heat capacity of grout and pipe into account. This model is validated to be accurate and has been implemented as part of a component model of the TRNSYS, but its numerical calculation is sophisticated and time-consuming.

Considering the axial heat flow and taking the long-term effect of the limited borehole depth into account, a 2-D finite line source model was established by Zeng et al. (2002) to analyze the heat transfer outside vertical borehole GHE. This 2-D model assumes the ground as a homogeneous semi-infinite medium with a uniform initial temperature, and assumes the borehole as a line source with finite length releasing heat at a constant rate per length. Evolved from the vertical borehole systems, inclined boreholes are considered as a favorable alternative to further reduce the land areas required for the GHEs. Then a 3-D finite line source model for inclined borehole GHE was also proposed by Cui et al. (2006). In order to take characteristics of the pile GHEs into proper consideration and to deal with the short term temperature response, Man et al. (2010) proposed a "solid" cylindrical source model, which suppose that the cylinder is no longer a cavity, but filled with the medium identical to that out of the cylinder, so that the whole infinite domain is composed of a homogeneous medium.

\subsection{Vertical and inclined finite line source model}

As mentioned, the 2-D vertical finite line source model and the 3-D inclined finite line source model are established based on the Green's function method to analyze the heat conduction outside the vertical and inclined boreholes, as shown in Fig. 4. An analytical solution has been derived for the 3-D inclined finite line source model as follows (Cui et al., 2006). Then, the 2-D model of vertical finite line source becomes a special case of the 3-D model with $\alpha=0$.

$$
t-t_{0}=\frac{q_{l}}{4 \pi k} \int_{0}^{H}\left(\frac{\operatorname{erfc}\left(\frac{r_{+}}{2 \sqrt{a \tau}}\right)}{r_{+}}-\frac{\operatorname{erfc}\left(\frac{r_{-}}{2 \sqrt{a \tau}}\right)}{r_{-}}\right) d S
$$

where

$$
\begin{aligned}
& r_{+}=\sqrt{\left(x-x_{0}-s \sin \alpha \cos \beta\right)^{2}+\left(y-y_{0}-s \sin \alpha \sin \beta\right)^{2}+(z-s \cos \alpha)^{2}} \\
& r_{-}=\sqrt{\left(x-x_{0}-s \sin \alpha \cos \beta\right)^{2}+\left(y-y_{0}-s \sin \alpha \sin \beta\right)^{2}+(z+s \cos \alpha)^{2}} .
\end{aligned}
$$

\subsection{Infinite and finite solid cylindrical source model}

For the solid cylindrical source model, a heat source shaped in a cylindrical surface of a radius $r_{0}$ is supposed to be buried in the medium with its axis being coincident with $z$ axis. 


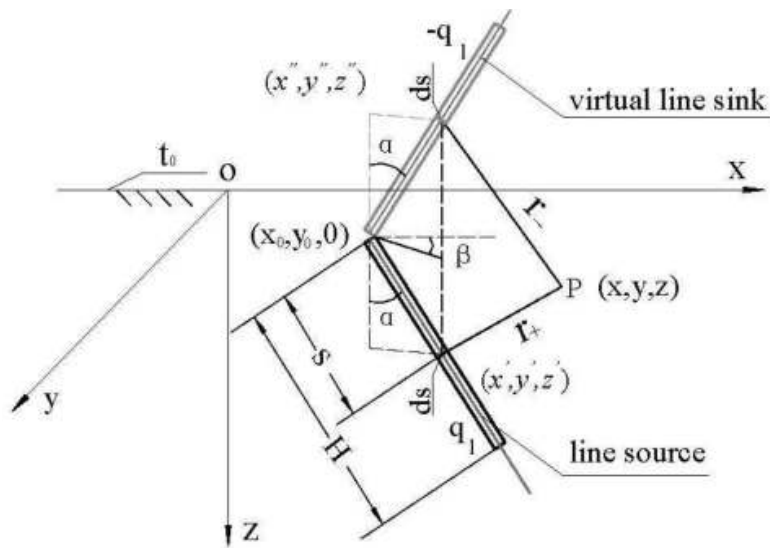

Fig. 4. Schematic diagram of an inclined finite line source in a semi-infinite medium

\subsubsection{The Infinite solid cylindrical source model}

First, the 1-D infinite solid cylindrical source model is studied with the axial heat flow neglected (Man et al., 2010). The analytical solution of this 1-D problem can be obtained directly with the Green's function method. The cylindrical heat source can be regarded as a collection of numerous line sources disposed along a circle of the radius $r_{0}$. The temperature rise at any location with the radial coordinate $r$ should be the sum, or integral, of all the individual temperature rises caused by the corresponding line sources. Then the analytical solution of the infinite solid cylindrical source model can be written as:

$$
\theta_{1}(r, \tau)=-\frac{q_{l}}{4 \pi \mathrm{k}} \int_{0}^{\pi} \frac{1}{\pi} \operatorname{Ei}\left(-\frac{r^{2}+r_{0}^{2}-2 r r_{0} \cos \varphi}{4 a \tau}\right) d \varphi
$$

Alternatively, the cylindrical heat source in this model can be reckoned as a collection of numerous ring line heat sources piled along the axial direction. Induced by a single instant ring line source with radius $r=r_{0}$ which lies on the plane $z=z^{\prime}$ and releases heat at the instant $\tau^{\prime}$, the temperature rise at any location of the coordinate $(r, z)$ and at the instant $\tau$ can be obtained according to the Green's function theory as:

$$
\theta^{*}=\frac{Q}{\rho c} \frac{1}{8\left[\sqrt{\pi a\left(\tau-\tau^{\prime}\right)}\right]^{3}} \exp \left[-\frac{r^{2}+r_{0}{ }^{2}+\left(z-z^{\prime}\right)^{2}}{4 a\left(\tau-\tau^{\prime}\right)}\right] I_{0}\left[\frac{r r_{0}}{2 a\left(\tau-\tau^{\prime}\right)}\right]
$$

Thus, the overall temperature rise caused by the infinite cylindrical heat source at any location should be the sum, or integral, of all the individual temperature rises caused by the corresponding ring sources over the duration from 0 and $\tau$. Then, the solution of the infinite solid cylindrical source model can be written, alternatively, as:

$$
\theta_{1}(r, \tau)=\frac{q_{l}}{\rho c} \int_{0}^{\tau} \int_{-\infty}^{\infty} \frac{1}{8\left[\sqrt{\pi a\left(\tau-\tau^{\prime}\right)}\right]^{3}} \exp \left[-\frac{r^{2}+r_{0}{ }^{2}+z^{\prime 2}}{4 a\left(\tau-\tau^{\prime}\right)}\right] I_{0}\left[\frac{r r_{0}}{2 a\left(\tau-\tau^{\prime}\right)}\right] d z^{\prime} d \tau^{\prime}
$$


This solution is independent of the axial coordinate $z$, and it provides a means to tackle the relevant finite problem.

\subsubsection{The finite solid cylindrical source model}

A 2-D finite solid cylindrical source model has also been presented (Man et al., 2010) in order to consider the influences of the finite length of the cylindrical heat source and the boundary. Similar to the approach for the finite line source problem, a virtual cylindrical sink with the same length but a negative heating rate may be set on symmetry to the boundary. Then, the solution of this problem can be obtained as:

$$
\begin{aligned}
& \theta_{2}(r, z, \tau)=\frac{q_{l}}{\rho c} \int_{0}^{h} d z^{\prime} \int_{0}^{\tau} \frac{1}{8\left[\sqrt{\pi a\left(\tau-\tau^{\prime}\right)}\right]^{3}} I_{0}\left[\frac{r r_{0}}{2 a\left(\tau-\tau^{\prime}\right)}\right] . \\
& \left\{\exp \left[-\frac{r^{2}+r_{0}{ }^{2}+\left(z-z^{\prime}\right)^{2}}{4 a\left(\tau-\tau^{\prime}\right)}\right]-\exp \left[-\frac{r^{2}+r_{0}{ }^{2}+\left(z+z^{\prime}\right)^{2}}{4 a\left(\tau-\tau^{\prime}\right)}\right]\right\} d \tau^{\prime}
\end{aligned}
$$

Temperature response at the half depth of the cylinder surface calculated with the infinite and finite solid cylindrical source models are compared in Fig. 5. Clear distinction can be seen between the responses of these two models. While the temperature response rises continuously with time for the infinite model, the temperatures of those GHEs simulated by the finite model with different finite lengths tend to produce different steady-state temperatures as time approaches infinity. This feature indicates that, it is important to take the finite length effect of heat source into account and to utilize the finite solid cylindrical source model in simulating the long-term operation of the GHE.

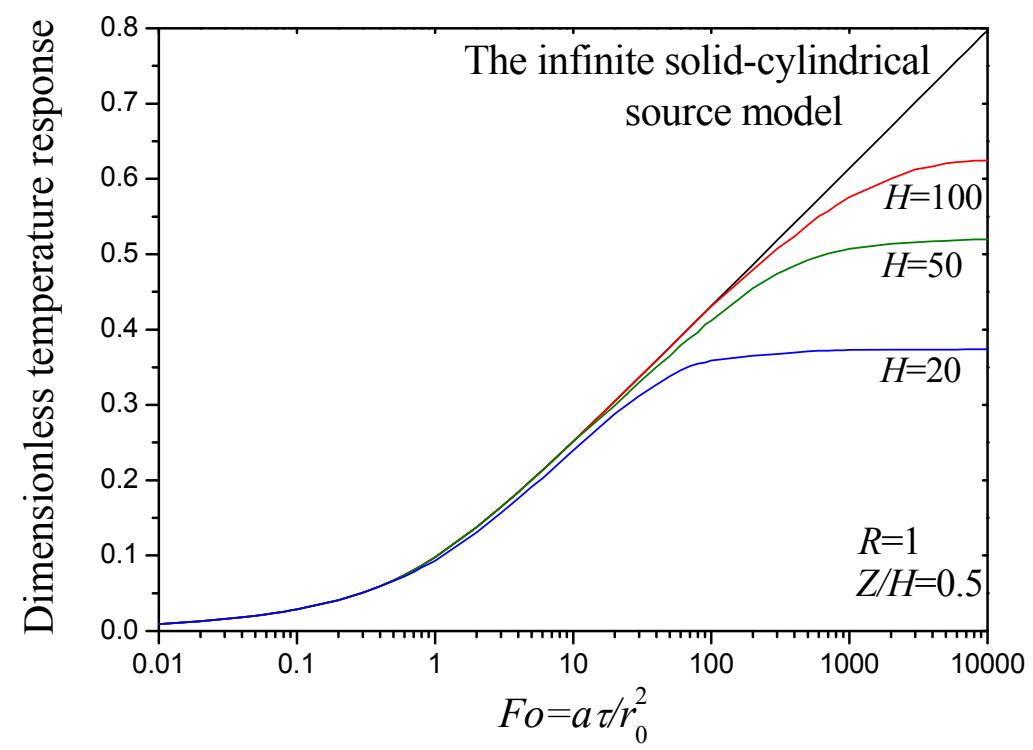

Fig. 5. Temperature response vs. time from infinite and finite solid cylindrical source models 


\section{Heat transfer with groundwater infiltration}

In practice, the boreholes of GHEs may penetrate several geologic strata. Below the water table, water is held and moves between the grains of geologic formations in response to hydraulic gradients. In general, a moderate groundwater advection is expected to make notable difference in alleviating the possible heat buildup around the borehole over time. As a result, it is desirable to account for the groundwater infiltration in the heat transfer model to avoid over-sizing of the GHEs. However, all of the GHE design tools available at present are based simply on principles of heat conduction, and do not consider the implications of groundwater flow in carrying away heat due partly to lack of appropriate analytical tools.

Taking the groundwater advection into account, the combined heat transfer models of conduction and advection in the GHE have been solved with analytical approach. Explicit expressions about temperature response of the moving infinite/finite line source models have been derived (Diao et al., 2004; Nelson et al., 2011). In these studies the ground around the boreholes is assumed to be a homogeneous porous medium saturated by groundwater. The groundwater velocity is uniform in the whole domain concerned and parallel to the ground surface. Heat is transported through the saturated porous medium in a combined mechanism: by conduction through its solid matrix and liquid in its pores as well as by convection of the moving liquid.

\subsection{Moving infinite line-source model}

The partial differential equation for advective and conductive heat transport in porous media can be expressed in a 2-D form ( $\mathrm{x}-\mathrm{y}$ plane) as follows (Domenico \& Schwartz, 1998):

$$
\rho c \frac{\partial T}{\partial t}+u_{x} \rho_{w} c_{w} \frac{\partial T}{\partial x}-\lambda\left(\frac{\partial^{2} T}{\partial x^{2}}+\frac{\partial^{2} T}{\partial y^{2}}\right)=0
$$

The solution of equation (15) for an infinite porous medium with a uniform initial temperature was given by Sutton et al. (2003) and Diao et al. (2004):

$$
\Delta T_{2}(x, y, t)=\frac{q_{L}}{4 \lambda \pi} \exp \left[\frac{v_{T} x}{2 a}\right]_{0}^{v_{T}^{2} t / 4 a} \frac{1}{\psi} \exp \left[-\psi-\frac{v_{T}^{2}\left(x^{2}+y^{2}\right)}{16 a^{2} \psi}\right] d \psi
$$

This analytical solution applies for the response of a constant line source with infinite length along the $z$-direction with a continuous heat flow rate per unit length of the borehole, $q_{L}$. Although a GHE is composed of a buried pipe that commonly is surrounded by grouting material, approximation by a line source is commonly accepted in heat transport models of GCHP systems (Diao et al., 2004; Eskilson, 1987; Sutton et al., 2003). The underground is assumed to be homogeneous with respect to the thermal and hydraulic parameters. For steady state conditions equation (16) becomes:

$$
\Delta T_{2 s}(x, y)=\frac{q_{L}}{2 \pi \lambda} \exp \left[\frac{v_{T} x}{2 a}\right] K_{0}\left[\frac{v_{T} \sqrt{x^{2}+y^{2}}}{2 a}\right]
$$


Introducing the dimensionless variable $P e=v_{T} H / a$ (Peclet number), equations (16) and (17) can be expressed in dimensionless forms:

$$
\begin{gathered}
\Theta_{2}(R, F o, P e)=\exp \left[\frac{P e}{2} R \cos (\varphi)\right]_{0}^{P e^{2} F o / 4} \frac{1}{\psi} \exp \left[-\psi-\frac{P e^{2} R^{2}}{16 \psi}\right] d \psi \\
\Theta_{2 s}(R, P e)=2 \exp \left[\frac{P e}{2} R \cos (\varphi)\right] K_{0}\left[\frac{P e}{2} R\right]
\end{gathered}
$$

The isotherms of a GHE field with groundwater advection simulated with the moving infinite line source model are shown in Fig. 6. Analysis on the influence of the groundwater advection on GHE performance may be found in details elsewhere (Diao et al., 2004).

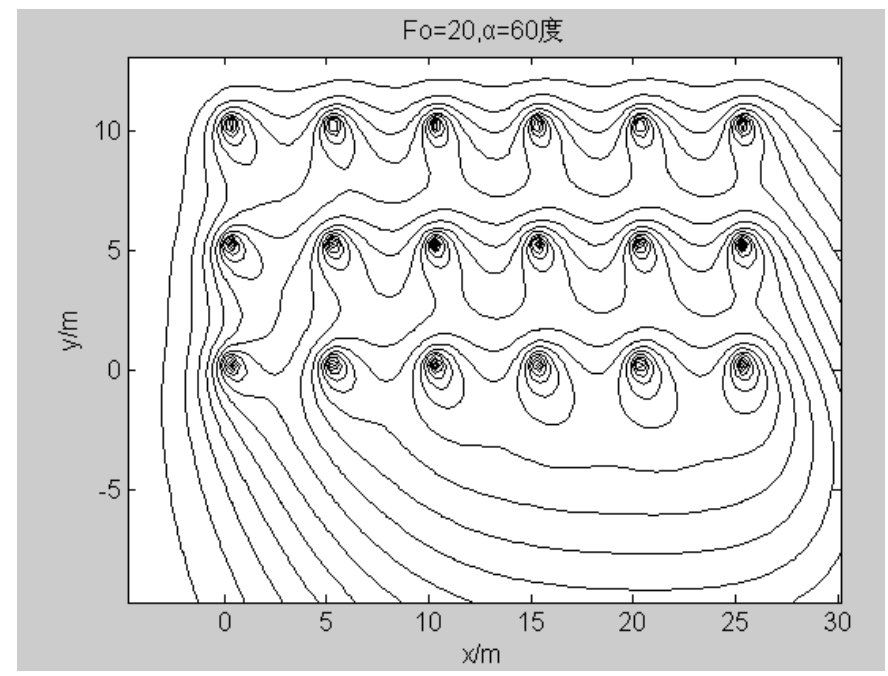

Fig. 6. Isotherms of a GHE field of 18 boreholes with groundwater advection

\subsection{Moving finite line-source model}

For long-term period simulations of the GHEs axial effects become more evident. Therefore, the moving finite line source model has been further established based on the Green's function by applying the method of images (Eskilson, 1987) and the moving source theory (Carslaw \& Jaeger, 1959). The detailed derivation is presented by Nelson et al. (2011). The transient solution reads as:

$$
\begin{gathered}
\Delta T_{3}(x, y, z, t)=\frac{q_{L}}{2 \lambda \pi} \exp \left[\frac{v_{T} x}{2 a}\right]\left[\int_{0}^{H} f(x, y, z, t) d z^{\prime}-\int_{-H}^{0} f(x, y, z, t) d z^{\prime}\right] \\
f(x, y, z, t)=\frac{1}{4 r}\left[\exp \left(-\frac{v_{T} r}{2 a}\right) \operatorname{erfc}\left(\frac{r-v_{T} t}{2 \sqrt{a t}}\right)+\exp \left(\frac{v_{T} r}{2 a}\right) \operatorname{erfc}\left(\frac{r+v_{T} t}{2 \sqrt{a t}}\right)\right]
\end{gathered}
$$


As time approaches infinity, the steady state solution is derived as follows:

$$
\Delta T_{3 s}(x, y, z)=\frac{q_{L}}{4 \lambda \pi} \exp \left[\frac{v_{T} x}{2 a}\right]\left[\int_{0}^{H} \frac{1}{r} \exp \left[-\frac{v_{T} r}{2 a}\right] d z^{\prime}-\int_{-H}^{0} \frac{1}{r} \exp \left[-\frac{v_{T} r}{2 a}\right] d z^{\prime}\right]
$$

Equations (20), (21) and (22) can be expressed in dimensionless forms:

$$
\begin{gathered}
\Theta_{3}(R, Z, F o, P e)=2 \exp \left[\frac{P e}{2} R \cos (\varphi)\right]\left[\int_{0}^{1} f(R, Z, F o, P e) d Z^{\prime}-\int_{-1}^{0} f(R, Z, F o, P e) d Z^{\prime}\right] \\
f(R, Z, F o, P e)=\frac{1}{4 R^{\prime}}\left[\exp \left(-\frac{P e}{2} R^{\prime}\right) \operatorname{erfc}\left(\frac{R^{\prime}-P e F o}{2 \sqrt{F o}}\right)+\exp \left(\frac{P e}{2} R^{\prime}\right) \operatorname{erfc}\left(\frac{R^{\prime}+P e F o}{2 \sqrt{F o}}\right)\right] \\
\Theta_{3 s}(R, Z, P e)=\exp \left[\frac{P e}{2} R \cos (\varphi)\right]\left[\int_{0}^{1} \frac{1}{R^{\prime}} \exp \left[-\frac{P e}{2} R^{\prime}\right] d Z^{\prime}-\int_{-1}^{0} \frac{1}{R^{\prime}} \exp \left[-\frac{P e}{2} R^{\prime}\right] d Z^{\prime}\right]
\end{gathered}
$$

Temperature contours obtained according to the moving finite and infinite line source models with groundwater advection considered are compared in Fig. 7. Note that temperature plumes are shorter for the finite model (Fig. 7a). Axial effects yields lower temperature changes at any given distance from the source due to the vertically dissipated heat. Temperature anomaly created in the vertical direction due to the axial effects can be observed in Fig. $7 \mathrm{~b}$. Obviously the differences between the models are most evident in the vicinity of the borehole ends.
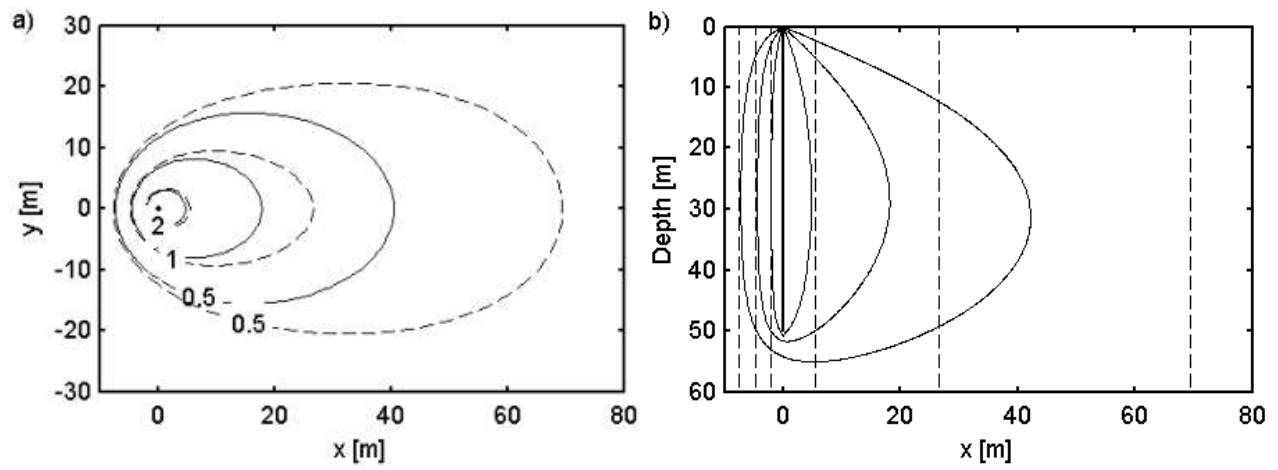

Fig. 7. Temperature contours $\left(\lambda=2.5 \mathrm{Wm}^{-1} \mathrm{~K}^{-1}, q_{l}=20 \mathrm{Wm}^{-1}, q=1.0 \times 10^{-7} \mathrm{~ms}^{-1}, t=20 \mathrm{yrs}\right)$. Solid lines: Moving finite line source model; Dashed lines: Moving infinite line source model. (a) Plan view (b) Vertical cross section.

By comparisons based on the simulation results, the shorter the borehole length is, the larger the discrepancy is between the moving finite and infinite line source models, and the shorter is the time when resulted temperature responses of the moving finite and infinite line source models start to differ. Besides, it is noticeable that the larger the Peclet number the lesser the discrepancy between the moving finite and infinite line source models. 


\section{Heat conduction around a buried spiral coil}

\subsection{Overview}

The GHE with vertical boreholes (Bose et al., 1985) has been the mainstream for the GCHP systems, which is also a major obstacle to apply the GCHP technology because its installation needs a substantial initial cost and requires additional ground area. In recent years foundation piles of buildings start to be utilized as part of the GHEs. These so-called "energy piles" combining the heat exchanger with building foundation piles are a notable progress in the GCHP applications, and its most competitive advantage is that it can reduce the initial cost as well as ground requirement for the borehole field.

Literature review has shown that most of existing studies of pile GHE were based on either experiments or numerical simulations (Morino \& Oka, 1994; Pahud et al., 1996; Pahud et al., 1999; Laloui et al., 2006; Hamada et al., 2007; Sekine et al., 2007). Besides, pipes are buried in concrete piles in configurations of U-tubes in most of such applications. The effective heat transfer area in a certain pile is limited, and air choking may occur in the turning tips of the tubes connected in series. In order to overcome these drawbacks, a novel configuration of the foundation pile GHE with a spiral coil has been proposed (Man et al., 2010). The distinct advantage of this novel GHE is that it can offer higher heat transfer efficiency, reduce pipe connection complexity, and decrease the thermal "short-circuiting" among the feed and return pipes. The schematic diagrams of a conventional single U-tube vertical borehole GHE and the pile GHE with spiral coil are compared in Fig. 8.
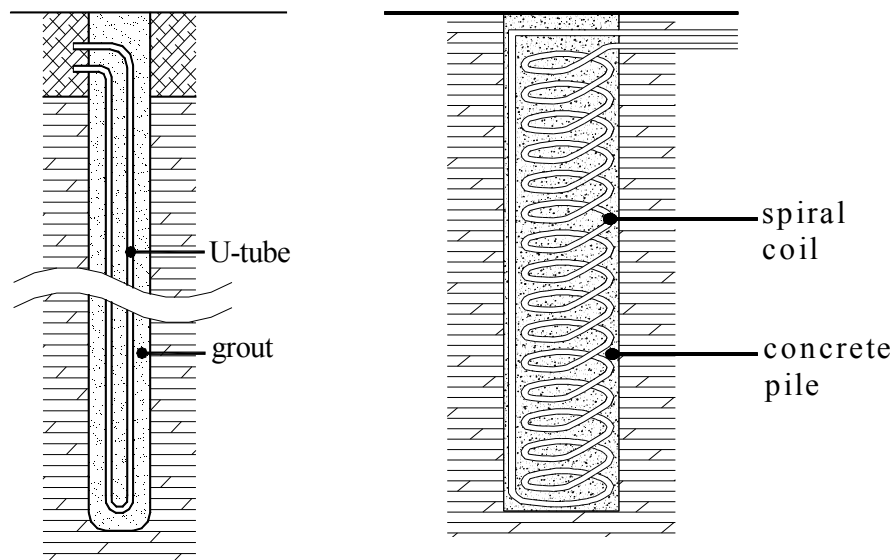

Fig. 8. Schematic diagram of a vertical borehole and a pile with a coil

Piles are much thicker in diameter but shorter in depth than boreholes. Obviously, either the line source models or the "hollow" cylindrical model mentioned in previous sections is no longer valid in this case. Due to its limited application history few analytical models on the buried spiral coils have been seen in literature. In order to better understand and simulate the heat transfer of buried spiral pipes, the authors have proposed two new kinds of models.

The first model is referred as the "ring-coil source model" (Cui et al., 2011), which is developed on the basis of cylindrical source model (Man et al., 2010), as shown in Fig. 9 (a) 
and (b). The ring-coil model further takes into account the discontinuity of the heat source and the impact of the coil pitch by simplifying the buried spiral coil as a set of separated rings located on the cylindrical surface. This model has made big progress from the classical models, however, the ring heat sources is discontinuous and separated with each other, which still deviates from the realistic conditions of the buried spiral coil.

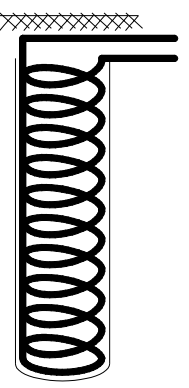

Pile GHE with spiral coil

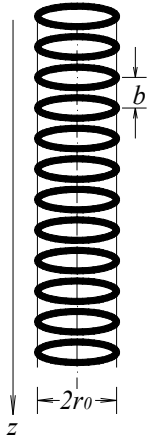

(a) Infinit ring-coil source model

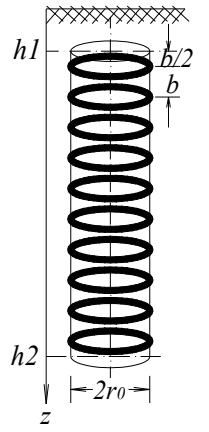

(b) Finit ring-coil source model

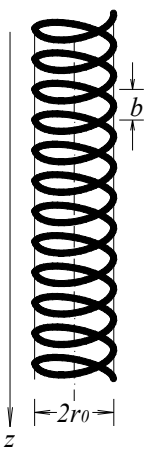

(c) Infinit spiral source model

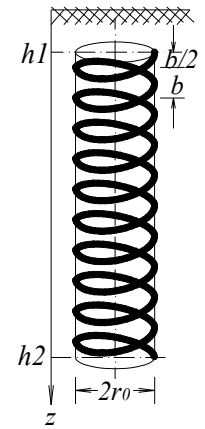

(d) Finit spiral source model

Fig. 9. Established heat source models of the pile GHE

Evolved from the ring-coil source model, the "spiral source model" (Man et al., 2011) is further presented for better analyzing and designing the pile GHE with spiral coils, as shown in Fig. 9 (c) and (d). For this model, the buried spiral coil is approximated by a spiral line heat source. The temperature response of the buried spiral coils can be evaluated according to the analytical solutions of this model.

\subsection{Ring model and spiral model}

\subsubsection{Ring-coil heat source model and solutions}

In the ring-coil heat source model, the buried spiral coil is simplified as a number of separated rings located on the cylindrical surface. In order to analyze the thermal effect of the heat transfer along the axis on the total heat transfer efficiency, both the infinite and finite ring-coil source models are discussed by means of the Green's function method.

To develop the analytical model of the buried spiral coils a basic and simple starting point is to study a single ring-coil heat source. Suppose the ring coil is located on the plane $z=z^{\prime}$ with its axis being coincident with $z$-axis with a continuous heating rate of $q$ since a starting instant, $\tau=\tau^{\prime}$. Based on the governing equation of transient heat conduction along with given boundary and initial conditions, the temperature response at location $(r, z)$ in the medium to such a single ring source can be obtained according to the Green's function theory:

$$
\Theta_{r, s}=\frac{1}{8 \pi^{3 / 2}} \int_{0}^{F o} \frac{1}{\left(F o-F o^{\prime}\right)^{3 / 2}} I_{0}\left[\frac{R}{2\left(F o-F o^{\prime}\right)}\right] \exp \left[-\frac{R^{2}+1}{4\left(F o-F o^{\prime}\right)}\right] \cdot \exp \left[-\frac{\left(Z-Z^{\prime}\right)^{2}}{4\left(F o-F o^{\prime}\right)}\right] d F o^{\prime}
$$




\subsubsection{Infinite ring-coil source model}

The infinite ring-coil source model is first studied, which means the ring-coil source is assumed to be infinite in the longitudinal direction. Define that the $z$-coordinates of the ring coils are $z^{\prime}= \pm(n+0.5) b$, where $\mathrm{n}=0,1,2, \ldots,+\infty$. As a consequence, the overall temperature response at a random point in the medium to all the ring sources can be determined as the sum of all the individual temperature rises caused by each ring-coil source:

$$
\begin{gathered}
\Theta_{r, i}(R, Z, F o)=\frac{B}{8 \pi^{3 / 2}} \sum_{n=0}^{\infty} \int_{0}^{F o} \frac{1}{\left(F o-F o^{\prime}\right)^{3 / 2}} I_{0}\left[\frac{R}{2\left(F o-F o^{\prime}\right)}\right] \exp \left[-\frac{R^{2}+1}{4\left(F o-F o^{\prime}\right)}\right] \\
\left\{\exp \left[-\frac{(Z-n B-0.5 B)^{2}}{4\left(F o-F o^{\prime}\right)}\right]+\exp \left[-\frac{(Z+n B+0.5 B)^{2}}{4\left(F o-F o^{\prime}\right)}\right]\right\} d F o^{\prime}
\end{gathered}
$$

\subsubsection{Finite ring-coil source model}

The infinite model neglects the effects of heat flow through the top and bottom ends of the heat source; therefore it is inadequate for the long-term operation of the GHE made of buried spiral coil. While keeping the ring-coil source simplification, the spiral coil is taken as a finite ring-coil source buried in a semi-infinite medium, stretching from $h_{1}$ to $h_{2}$ from the boundary of the ground surface. The coil is then approximated as $m$ pieces of rings. Again, the images of the ring coils with negative heating rate $-q_{l} b$ are set on symmetry to the boundary in order to keep the constant temperature of the ground surface, and the solution for the finite ring-coil source model is expressed as:

$$
\begin{aligned}
& \Theta_{r, f}(R, Z, F o)=\frac{B}{8 \pi^{3 / 2}} \int_{0}^{F o} \frac{1}{\left(F o-F o^{\prime}\right)^{3 / 2}} I_{0}\left[\frac{R}{2(F o-F o}\right] \exp \left[-\frac{R^{2}+1}{4(F o-F o}\right] . \\
& \sum_{n=0}^{m}\left\{\exp \left[-\frac{(Z-H 1-(n+0.5) B)^{2}}{4\left(F o-F o^{\prime}\right)}\right]-\exp \left[-\frac{(Z+H 1+(n+0.5) B)^{2}}{4\left(F o-F o^{\prime}\right)}\right]\right\} d F o^{\prime}
\end{aligned}
$$

\subsubsection{The spiral source model and its analytical solutions}

On the basis of the ring-coil source model, the spiral source model is further developed with increasing sophistication and accuracy to take the 3-D geometrical characteristic of spiral coil into account. In the spiral source model, the buried coil is represented by a spiral line heat source. Both the infinite and finite spiral source models are studied.

For an instantaneous point heat source with intensity of $\rho c$, located at $\left(r^{\prime}, \varphi^{\prime}, z^{\prime}\right)$ and activated at the instant $\tau^{\prime}$, its Green's function in the cylindrical coordinates at point $(r, \varphi, z)$ can be expressed as: 


$$
\begin{aligned}
& G\left(r, \varphi, z, \tau ; r^{\prime}, \varphi^{\prime}, z^{\prime}, \tau^{\prime}\right)=\frac{1}{8\left[\pi a\left(\tau-\tau^{\prime}\right)\right]^{3 / 2}} . \\
& \cdot \exp \left[-\frac{\left(r \cos \varphi-r^{\prime} \cos \varphi^{\prime}\right)^{2}+\left(r \sin \varphi-r^{\prime} \sin \varphi^{\prime}\right)^{2}+\left(z-z^{\prime}\right)^{2}}{4 a\left(\tau-\tau^{\prime}\right)}\right]
\end{aligned}
$$

\subsubsection{Infinite spiral source model}

The infinite spiral source model is discussed with the axial heat flow neglected, as shown in Fig. 9 (c). This spiral heat source can be considered as the sum, or integral, of numerous point heat sources located on the spiral line with the instantaneous intensity of $q_{l} b d \varphi^{\prime} d \tau^{\prime} /(2 \pi)$, which cylindrical coordinates keep $r^{\prime}=r_{0}, z^{\prime}=b \varphi^{\prime} /(2 \pi)$. Then the temperature response in the medium resulted from the step heating of the spiral heat source from the starting instant $\tau^{\prime}=0$ can be deduced according to the Green's function and superimposing theory based on the temperature response to a point heat source:

$\Theta_{i, \text { spiral }}=\frac{B}{16 \pi^{5 / 2}} \int_{0}^{F o}\left(\frac{1}{F o-F o^{\prime}}\right)^{3 / 2} \cdot \int_{-\infty}^{\infty} \exp \left[-\frac{R^{2}+1-2 R \cos \left(\varphi-\varphi^{\prime}\right)+\left(Z-B \varphi^{\prime} / 2 \pi\right)^{2}}{4\left(F o-F o^{\prime}\right)}\right] d \varphi^{\prime} d F o^{\prime}$

\subsubsection{Finite spiral source model}

In order to take the effects of heat flow through the top and bottom ends of pile into account and investigate the long-term operation performance of the pile GHE, the finite spiral source model is proposed and analyzed. In this model, the coil pipe buried in the pile is considered as a finite-length spiral coil in a semi-infinite medium, as shown in Fig. 9 (d).

With the virtual heat source theory, a virtual spiral heat sink with negative heating rate $-q_{l}$ and of identical physical dimensions is set on symmetry to the boundary. Then the finite spiral heat source and heat sink can be approximated as the sum of numerous point heat sources and heat sinks. Again, the Green's function theory is employed to obtain the temperature response of the medium. For the finite-length spiral source starts at $z^{\prime}=h_{1}$, or $\varphi^{\prime}=2 \pi h_{1} / b$, and ends at $\varphi^{\prime}=2 \pi h_{2} / b$ buried in a semi-infinite medium with a step heating rate per length of the pile, $q_{l}$, the solution may be derived:

$$
\begin{aligned}
& \Theta_{f, \text { spiral }}=\frac{B}{16 \pi^{5 / 2}} \int_{0}^{F o}\left(\frac{1}{F o-F o^{\prime}}\right)^{3 / 2} \cdot \exp \left[-\frac{R^{2}+1}{4\left(F o-F o^{\prime}\right)}\right] \cdot \\
& \int_{2 \pi H_{1} / B}^{2 \pi H_{2} / B} \exp \left[\frac{2 R \cos \left(\varphi-\varphi^{\prime}\right)}{4\left(F o-F o^{\prime}\right)}\right] \cdot\left\{\exp \left[-\frac{\left(Z-B \varphi^{\prime} / 2 \pi\right)^{2}}{4\left(F o-F o^{\prime}\right)}\right]-\exp \left[-\frac{\left(Z+B \varphi^{\prime} / 2 \pi\right)^{2}}{4\left(F o-F o^{\prime}\right)}\right]\right\} d \varphi^{\prime} d F o^{\prime}
\end{aligned}
$$

Take an example of buried spiral source with $B=1, H_{1}=2.0$, and $H_{2}=12.0$, the temperature distributions in the longitudinal profile of pile as well as the ground at the dimensionless time of $F_{o}=1.0$ calculated with infinite and finite spiral source model are compared in Fig. 10. In general, the spiral configuration of the heat source gets well representation. 
Dimensionless temperature rise $\Theta_{r, f}$

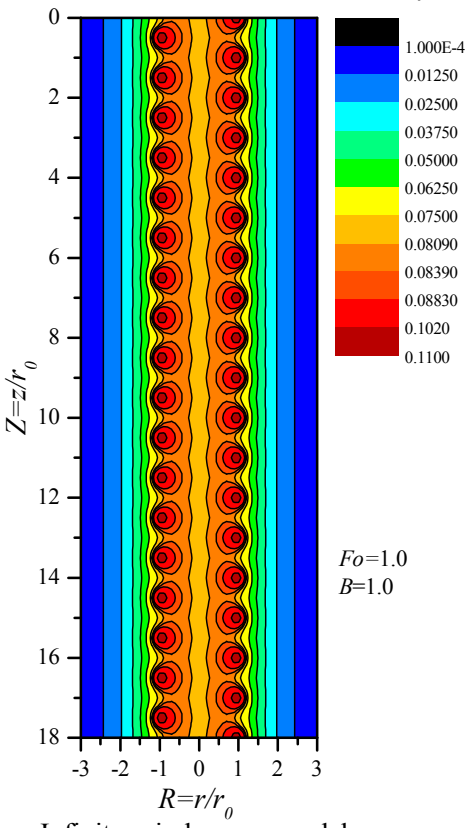

Infinite spiral source model
Dimensionless temperature rise $\Theta_{r, f}$

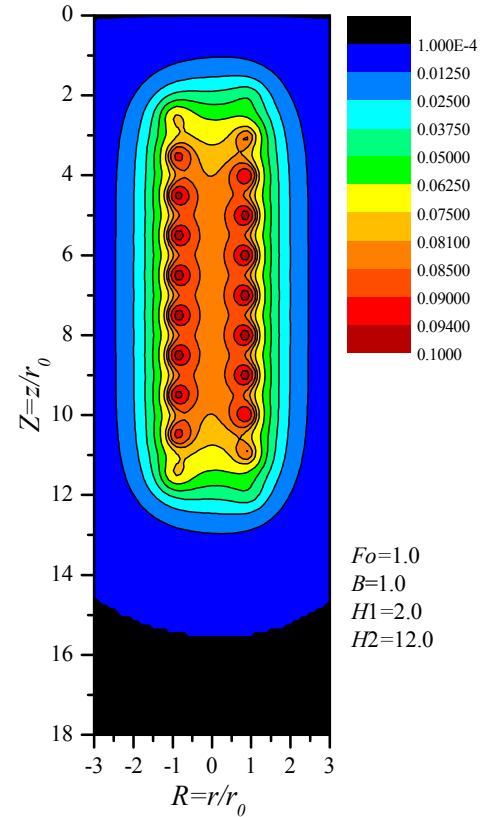

Finite spiral source model

Fig. 10. Temperature response of the infinite and finite spiral source models

The dimensionless temperature response at the midpoint of spiral heat source calculated with infinite and finite spiral source model are further compared in Fig. 11.

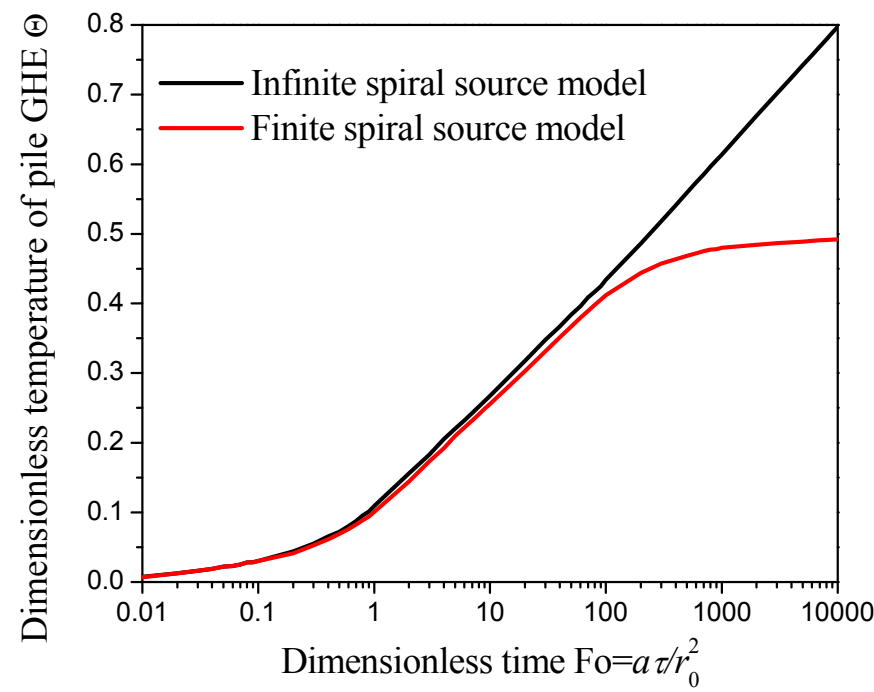

Fig. 11. Dimensionless temperature vs. time from infinite and finite spiral source models 
As shown in Fig. 11, the finite model yields a relatively lower temperature response compared with the infinite model. Temperature rises calculated with these two models are in good agreement for a short period after the start of heating. As time goes on, remarkable discrepancy of the finite model from the infinite one appears since the former takes the heat transfer through the top and bottom ends of the pile into account. While the temperature response rises continuously with time for the infinite model, temperature response for the finite model tends to a steady state as time approaches infinity. This feature indicates the importance to take the finite length effect into account in consideration of the long term operation of the buried spiral heat source. The heat transfer features of the pile GHE can be adequately described by the finite spiral source model, and its analytical solutions have provided a desirable tool for simulating the pile GHE and prompting its applications.

\section{Ground heat exchangers with multiple boreholes}

GHEs in practical GCHP projects usually consist of multiple boreholes. The conduction problems under the assumption of constant properties satisfy the condition of superposition; therefore the temperature rise at a certain location in the GHE with multiple boreholes can be obtained by means of summing up all the individual temperature excesses caused by each of the boreholes at the concerned spot, that is:

$$
\theta_{m}(\tau)=\sum_{i=1}^{N} \theta_{i}(\tau)
$$

According to simulation requirements and conditions the function for the temperature excess, $\theta_{i}(\tau)$, caused by a single borehole may be determined from models discussed in previous sections such as the finite line source model or cylindrical source model.

As mentioned above, while the superposition approach is employed for thermal analysis of GHEs, the domain involved is divided into two separate regions, i.e. the region inside the borehole and that outside it. For the former region the heat transfer is considered as steadystate. For heat transfer outside the borehole, transient heat transfer models should be used. The temperature on the interface of the two regions, i.e. the borehole wall, constitutes a key link of the thermal analyses in the two regions. The mean temperature of the circulating fluid, and, then its inlet and outlet temperatures, varying with time, can be determined with the borehole temperature plus a temperature difference resulted from the borehole resistance. The temperature on borehole wall, however varies along its depth as indicated in previous discussions, and differs from each other among different boreholes. As a consequence, it is desirable to define a representative temperature of the borehole wall for the entire borehole field so as to keep the analysis concise enough for engineering design and thermal analysis purposes.

Normally, the temperature response at the midpoint of borehole in depth-direction is selected to represent the borehole wall temperature response for each individual borehole. Although more sophisticated approaches have been investigated such as taking the integrated average temperature along the borehole depth as the representative one, the study (Zeng et al. 2003) has shown that the simpler choice of the midpoint temperature as the representative one is acceptable for engineering applications. 
On determination of the temperature rise on a certain borehole wall it is important to distinguish the temperature rise caused by the heat source (U-tubes) in the borehole itself, which is usually the most significant, and those caused by thermal interference from other boreholes in the GHE. The spaces between adjacent boreholes are much greater than borehole radius, as a consequence, the minor discrepancy in the temperature rises on the borehole perimeter in the circumferential direction caused by an adjacent borehole can be neglected, and, then, the distance between the two borehole axes is counted. As a result, for calculation of the temperature rise on a borehole wall equation (32) turns to be:

$$
\theta_{m}(\tau)=\theta\left(r_{b}, 0.5 H, \tau\right)+\sum_{j=1}^{N-1} \theta\left(x_{j}, 0.5 H, \tau\right)
$$

where $x_{\mathrm{j}}$ is the distance between the $j$ borehole and the borehole concerned.

In the GHE with multiple boreholes the boreholes experience diversified temperature responses owing to their specific locations in the GHE configuration and, then, different heat transfer conditions. A representative borehole needs to be selected to determine the temperature rise on the borehole wall to avoid too large a workload of computation. It is usually recommended for engineering design to take the least favorable borehole as the representative, i.e. the one with largest temperature rise in the GHE. It is defined as:

$$
\theta_{e}=\operatorname{Max}\left(\theta_{m, i}\right)=\operatorname{Max}\left(\theta_{i}+\sum_{\substack{j=1 \\ j \neq i}}^{N} \theta_{i j}\right)
$$

It is easy to locate the least favorable borehole in most of the GHE configurations for pure conduction models; and this choice is conservative for GHE design. While ensuring safe operation of the GHE, it leads to over-sizing of the GHE and aggravating its cost. In view of fact that larger and larger GHEs are constructed consisting of hundreds boreholes in a single GHE, this choice of the representative borehole can result in too severe deviations. A desirable alternative for the representative borehole would be the one whose temperature rise follows closely the average temperature rise of the GHE. This task is demanding even for the pure conduction models due to the wide diversity of possible configurations of the GHE. Some studies are under the way on this subject, and the borehole locating at the nearest vicinity of the geometric center of a quarter of a matrix configuration of the GHE is considered to be an appropriate choice for the representative one in pure conduction models (Lin, 2010). For the advection models it is even more intricate to find out a proper representative borehole because another factor, the velocity of the groundwater infiltration is incorporated into the model while its direction has added more numerous variations relative to the orientation of the GHE configuration. This seems a problem which needs to be addressed to properly in such an approach.

\section{Temperature response to variable loads}

GCHP systems can provide buildings with heating and cooling in different seasons, so heat can be extracted from or rejected to the ground. As defined, the heat load $q_{1}$ means the heat 
rejected to the ground, so the temperature rise in ground is positive; they both turn to negatives if heat is extracted from the ground.

In order to analyze the intricate heat transfer process in the GHEs effectively and efficiently, a basic and simple model must be established and solved first, and then, more complicated factors are added gradually. The basic problem is the heat transfer of a single borehole under a step heating, which means a constant heating rate starting from a certain instant. All the models for outside boreholes heat transfer discussed in previous sections deal with the primary problem of step heating.

The heat extracted from or rejected to the ground varies with time because the GCHP load usually varies with time. The variable heat flow can be approximated by a pulse train of heating load as shown in Fig. 12. A heating pulse imposed in the time interval $\tau_{i}<\tau<\tau_{i+1}$ can be considered as superposition of two step heating fluxes, as shown in Fig. 13.

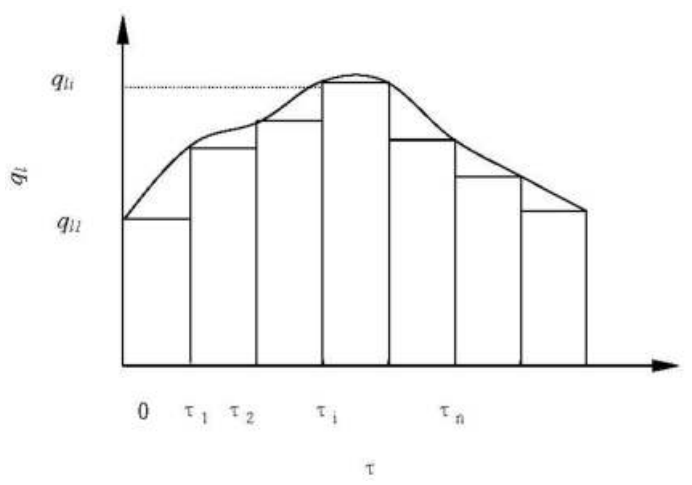

Fig. 12. Continuous heating approximatedby a rectangular pulse train

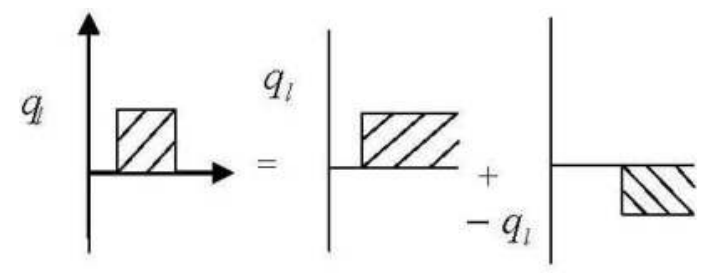

Fig. 13. A pulse heating equals two step heating fluxes

In order to facilitate computation of the temperature response of the GHEs to such sequential heating pulses the concept of so-called $g$-function is usually introduced. The $g$ function represents the non-dimensional temperature response on the representative borehole wall to the step heating for a specific configuration of the GHE, which is defined as

$$
g(\tau)=\frac{2 \pi k \theta_{e}}{q_{l}}
$$


On the assumption of a uniform heating rate $q_{1}$ in all the boreholes of the GHE, the $g$ function is independent of $q_{1}$. The temperature response to the step heating on the representative borehole wall can be determined on basis of the models presented in previous sections together with the superposition procedures such as that of equation (34).

For sequential heating pulses shown in Fig. 12, the borehole wall temperature rise at time $\tau$ can be obtained by superposition as:

$$
\theta=\frac{1}{2 \pi k} \sum_{i=1}^{\infty}\left(q_{l_{i}}-q_{i_{i-1}}\right) \cdot g\left(\tau-\tau_{i}\right), \quad\left(q_{l_{0}}=0\right)
$$

Let's consider a simple case of periodic on-off operation of the GCHP system to demonstrate the impact of discontinuous heating. Assume that the discontinuous GCHP operation is cyclic with a period $T$, of which the on-time is $T_{1}$. Then, $C=T_{1} / T$ denotes the on-time ratio. If the average heating intensity over the operating period is denoted by $\overline{q_{l}}$, the pulse heating intensity is $\overline{q_{l}} / C$, as shown in Fig. 14 (a).

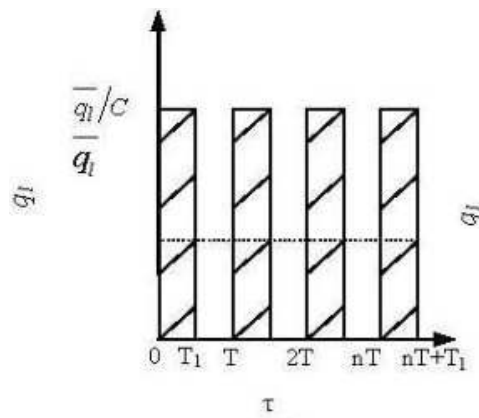

(a)

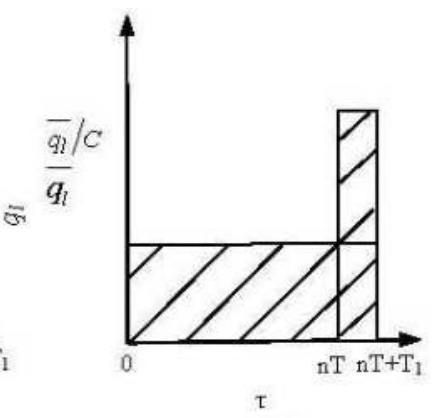

(b)

Fig. 14. A cyclic pulse load and its simplification

The borehole wall temperature response in a single borehole GHE to such cyclic pulse heating is calculated based on the line source model. Fig. 15 shows the borehole wall temperature response with the same average heating intensity but different operating time ratios. Simulations indicate that on such conditions the borehole wall temperature oscillates significantly while rising gradually over cycles. Furthermore, the smaller the operating time ratio is, which corresponds to stronger pulse intensities, the larger temperature swings are resulted in. The maximum temperature rise of the fluid after an operating period is an important criterion in design and thermal analysis of the GHEs. Study has shown that the maximum borehole wall temperature rise due to the periodic on-off heating load can be approximated by superposition of temperature rises caused by a continuous mean load and a single heating pulse as shown in Fig. 14 (b). Fig. 16 shows that the maximum temperature rise obtained from the simplified model is equivalent to that from the exact periodical pulse load model. Thus, this simplification provides an approach to analyze discontinuous loads over long durations. The results also indicate that the maximum temperature rise depends not only on the mean load over the whole duration, but also on the intensity and operating span of a single pulse. 


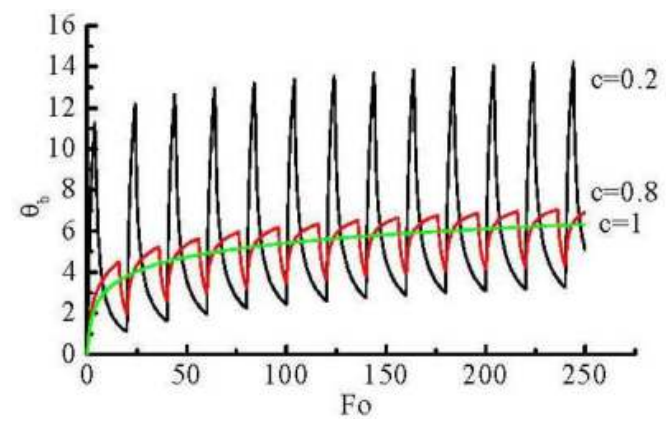

Fig. 15. Borehole wall temperature response to cyclic pulse loads

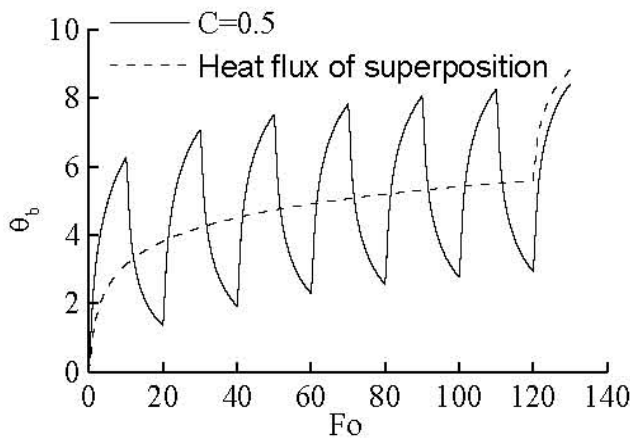

Fig. 16. Temperature response to a cyclic load and its simplification

\section{Design and simulation software for ground heat exchangers}

As mentioned above, the heat transfer process in a GHE involves quite a number of factors. It is necessary to further develop an accurate, reliable and convenient program for GHE design and simulation. In the last decade, a number of GHE models have been developed and they have been combined, directly or indirectly, with models of the building, heat pumps, and other components in various modeling environments such as TRNSYS, EnergyPlus, eQuest, and HVACSIM+. The GHE model used in TRNSYS (Hellström, 1989) is called the Duct Ground Heat Storage model, originally intended for underground thermal storage systems. The model uses numerical solutions for the global heat transfer between the storage volume and the far-field, and for the local problem of the heat transfer around the boreholes. An analytical method is employed to solve the steady-flux problem around the nearest pipe. The three models implemented in HVACSIM+ (Xu \& Spitler, 2006), EnergyPlus (Fisher, 2006) and eQuest (Liu, 2008) have a common heritage, which are based on extensions of Eskilson's model (1987). The programs are based on pre-computed response functions for specific GHE geometries.

On basis of the study on the heat transfer modeling of the GHEs, a software package in Chinese interface named GeoStar has been developed and spread for the design and simulation of the GHEs mainly in China (Fang et al., 2002). This software package is able to size GHEs to meet the user-specified minimum and maximum entering fluid temperatures 
to a heat pump for a given set of design conditions, such as building load, ground thermal properties, borehole configuration, and heat pump operating characteristics. The heat transfer models mentioned above are employed in the software, including the analytical solution of the finite line source model for the thermal resistance outside boreholes and the quasi 3-D model for the thermal resistance inside boreholes. In addition, the modeling procedure uses spatial superimposition for multiple boreholes and sequential temporal superimposition to dealing with the dynamic heating and cooling loads of the systems. The flow chart of the computing procedure for the model implementation is described in Fig. 17. The design process is actually a simulation-based process by means of the trial-and-error method.

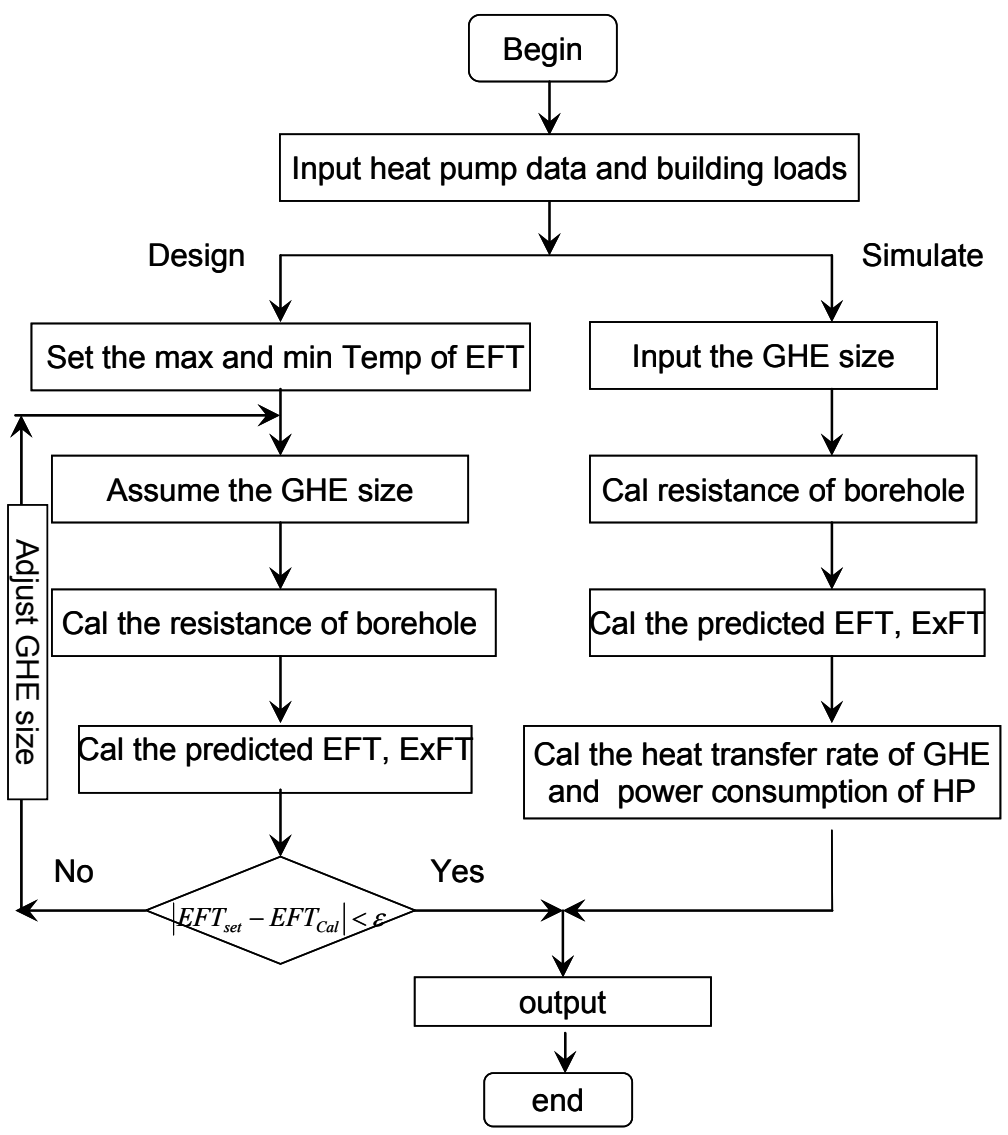

Fig. 17. The flowchart of the GeoStar program

The program with a friendly interface and visual graph has been developed under the Delphi Environment. In the visual interface, all the geometry parameters and inlet conditions can be set up in dialog boxes which can be popped up by clicking the different pages, as shown in Fig. 18. When all the required parameters are set up, the pre-compiled program will begin to simulate or design under the specific conditions. 


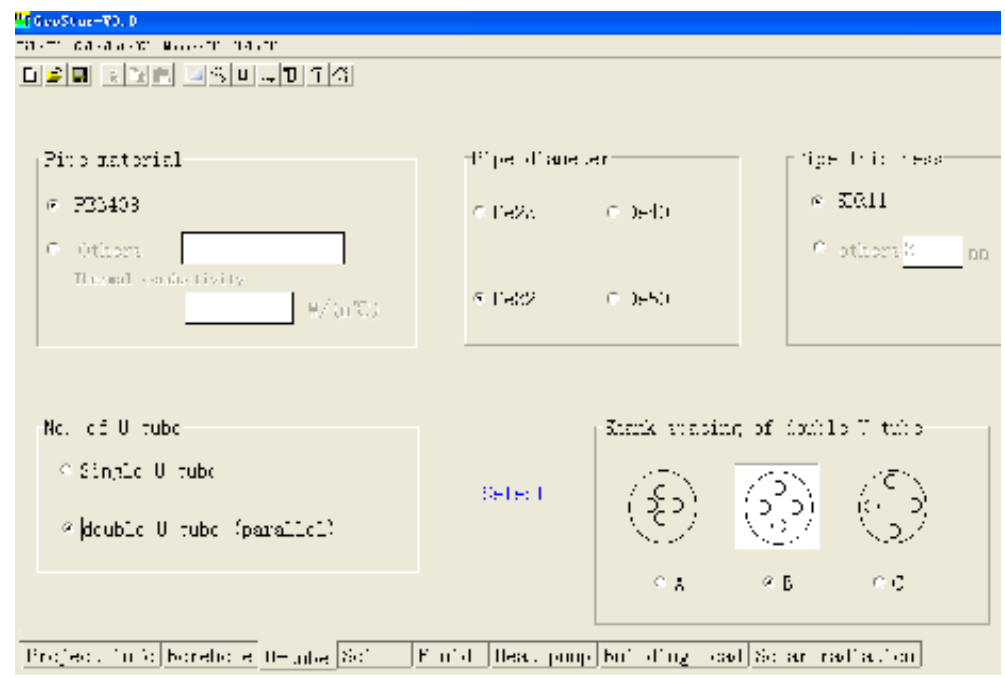

Fig. 18. Wizard user interface

In the past ten years a number of GHE models have been developed for use with various building simulation programs. In view of this, one of the authors together with other researchers conducted a comparison of GHE models developed for use with programs, including GeoStar, TRNSYS, HVACSIM+, GEOEASE II and eQuest (Spitler et al., 2009). The experimental validation was also carried out between the models and the experimental results. One of the research results is described in Fig. 19, which illustrated the predicted monthly average borehole ExFT and the measured data, when the hourly heat transfer rate was specified. It can be seen that all of the models (including GeoStar) predicted the ExFT within $1^{\circ} \mathrm{C}$, except HVACSIM+ which overpredicted a maximum of $2^{\circ} \mathrm{C}$, for the summer cooling months during the first year. For the shoulder seasons and heating months, the errors decreased slightly.

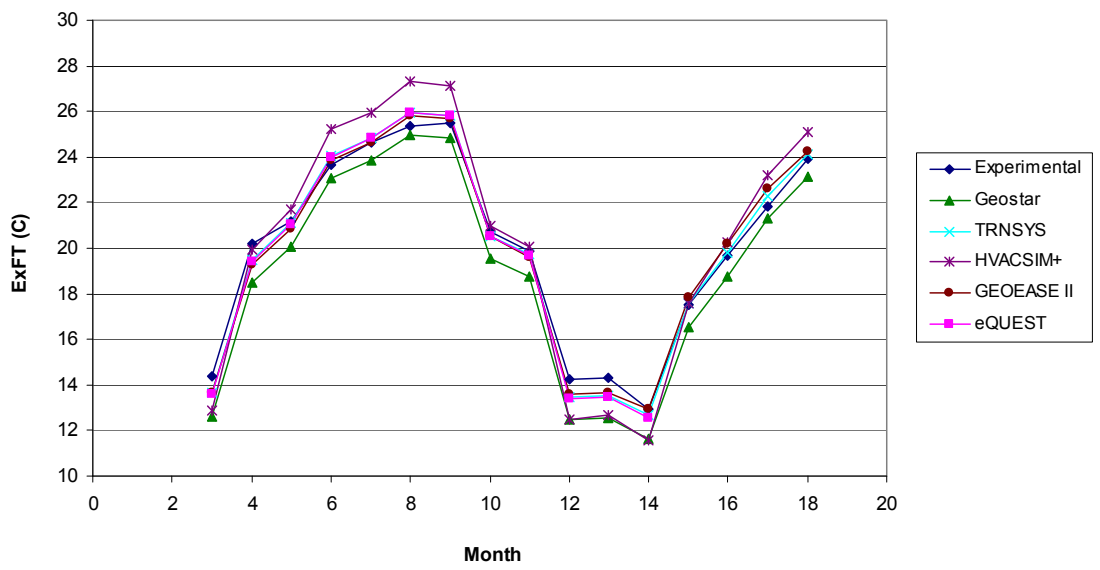

Fig. 19. Comparisons of experimental and predicted monthly average borehole ExFTs 


\section{Conclusion}

This chapter presents a comprehensive review of the study on heat transfer modeling of the ground heat exchangers in ground-coupled heat pump systems by means of superposition principle and analytical solutions. An entire set of techniques are provided to develop computer software for thermal analysis and design of GHEs in practical engineering.

Contributions of our research group to improvement of the GHE heat transfer modeling are expounded in particular, which include mainly

1. A quasi 3-D model for heat transfer inside borehole has been proposed, and its analytical solution derived to account for the borehole geometry and thermal interference among the legs of the U-tube.

2. The explicit analytical solutions of 2-D and 3-D models for vertical and inclined finite line source model for heat transfer outside borehole have been obtained to consider the axial heat flow and take the long-term effect of the limited borehole depth into account.

3. A solid cylindrical source model has been developed which gives a better description of the short-term temperature response for boreholes than the traditional 1-D models do, and may also serve as a tool for the pile GHE thermal analysis.

4. The advection models of infinite and finite line source have been proposed, and their analytical solutions derived to deal with the combined conductive and convective heat transfer in GHEs with groundwater infiltration taken into account.

5. The ring-coil source model and the spiral source model established for the heat transfer of a buried spiral coil to simulate heat transfer in the foundation pile GHE, which having created a new frontier of the GCHP applications.

The entire modeling uses the techniques of spatial superimposition for multiple boreholes and sequential temporal superimposition for arbitrary heating/cooling loads of the systems. The heat transfer models for the borehole GHE have been incorporated into a computer program, developed by our research group for providing a reliable and useful tool to design and simulate the GHE of GCHP systems. These studies on GHE heat transfer modeling in this chapter is expected to provide supports for developing the technique and promoting applications of the GCHP systems.

\section{References}

Bose, J. E.; Parker, J. D. \& McQuiston, F. C. (1985). Design/data manual for closed-loop ground coupled heat pump systems, Oklahoma State University for ASHRAE.

Carslaw, H. S. \& Jeager, J. C. (1947). Conduction of heat in solids, Oxford Press, Oxford.

Carslaw, H. S. \& Jaeger, J. C. (1959). Conduction of Heat in Solids, second ed. Oxford University Press, New York.

Cui, P; Yang, H. X.; \& Fang, Z. H. (2006). Heat transfer analysis of ground heat exchangers with inclined boreholes. Applied Thermal Engineering, 2006, 26: pp. 1169-1175. 
Cui, P.; Li, X.; Man, Y. \& Fang, Z. H. (2011). Heat transfer analysis of pile geothermal heat exchangers with spiral coils, Applied Energy, 2011, 88 : pp. 4113-4119.

Diao, N. R.; Li, Q. Y. \& Fang, Z. H. (2004). Heat Transfer in Ground Heat Exchangers with Groundwater Advection, International Journal of Thermal Sciences, 2004, 43 (12): pp. 1203-1211.

Diao, N. R. \& Fang, Z. H. (2006). Ground-coupled heat pump technology, The Higher Education Press, Beijing.

Domenico, P.A. \& Schwartz, F.W. (1998) Physical and Chemical Hydrogeology, seconded. John Wiley \& Sons Inc, New York.

Eskilson, P. (1987). Thermal analysis of heat extraction boreholes, Doctoral Thesis, University of Lund, Department of mathematical Physics, Lund, Sweden.

Fang, Z. H.; Diao, N. R. \& Cui, P. (2002). Discontinuous operation of geothermal heat exchangers. Tsinghua Science and Technology, 2002, 7(2): pp. 194-197.

Fisher, D. E.; Murugappan, A.; Padhmanabhan, S. K. \& Rees, S. J. (2006). Implementation and Validation of Ground-Source Heat Pump System Models in an Integrated Building and System Simulation Environment. HVAC\&R Research, 2006, 12 (3a): pp. 693-710.

Hamada, Y.; Saitoh, H.; Nakamura, M.; Kubota, H. \& Ochifuji, K. (2007). Field performance of an pile GHE system for space heating, Energy and Buildings, 2007: 39 (5): pp. 517-524.

Hellström, G. (1989). Duct Ground Heat Storage Model, Manual for Computer Code, Department of Mathematical Physics, University of Lund, Sweden.

Hellström, G. (1991). Ground heat storage, Thermal analysis of duct storage systems. Doctoral Thesis, Department of Mathematical Physics, University of Lund, Lund, Sweden.

Ingersoll, L. R. \& Zobel, O. J. (1954). Heat conduction with engineering and geological applications, 2nd edition, McGraw-Hill, New York.

Kavanaugh, S. P. (1997). Ground source heat pumps, Design of geothermal systems for commercial and institutional buildings. American Society of Heating, Refrigerating and Air-Conditioning Engineers (ASHRAE).

Laloui, L.; Nuth, M. \& Vulliet, L. (2006). Experimental and numerical investigations of the behaviour of a heat exchanger pile, International Journal for Numerical and Analytical Methods in Geomechanics, 2006, 30 (8): pp. 763-781.

Lin Y., (2010). Study on heat transfer modeling and design of ground heat exchangers, Master's Thesis, Shandong Jianzhu University, Jinan, China.

Liu, X. (2008). Enhanced design and energy analysis tool for geothermal water loop heat pump systems. Proceedings of 9th International Energy Agency Heat Pump Conference. pp. 20-22, May. Zürich, Switzerland.

Man, Y.; Yang, H. X. \& Fang, Z. H. (2008). Study on hybrid ground-coupled heat pump systems. Energy and Buildings, 2008, 40(11): pp. 2028-2036.

Man, Y.; Yang, H. X.; Diao, N. R.; Liu, J. H. \& Fang, Z. H. (2010). A new model and analytical solutions for borehole and pile ground heat exchangers. International Journal of Heat and Mass Transfer, 2010, 53: pp. 2593-2601. 
Man, Y.; Yang, H. X.; Li, X.; Cui, P.; \& Fang, Z. H. (2011). The spiral heat source model for the pile ground heat exchangers, In press by HVAC\&R Research (2011).

Mei, V. C. \& Baxter, V. D. (1986). Performance of a ground-coupled heat pump with multiple dissimilar U-tube coils in series. ASHRAE Transactions, 1986, 92(2): pp. 22-25.

Morino, K. \& Oka, T. (1994). Study on heat exchanged in soil by circulating water in a steel pile, Energy and Buildings, 1994, 21 (1): pp. 65-78.

Nelson, M. G.; Philipp, B.; Zhu, K.; Bayer, P. \& Fang, Z. H. (2011). A moving finite line source model to simulate borehole heat exchangers with groundwater advection, International Journal of Thermal Sciences, 2011, 50: pp. 2506-2513.

Pahud, D.; Fromentin, A. \& Hadorn, J. C. (1996). The Duct Ground Heat Storage Model (DST) for TRNSYS Used for the Simulation of Heat Exchanger Piles, DGC-LASEN, Lausanne.

Pahud, D.; Fromentin, A. \& Hubbuch, M. (1999). Heat exchanger pile system for heating and cooling at Zurich Airport, IEA Heat Pump Centre Newsletter, 1999, 17 (1): pp. 1516.

Sekine, K.; Ooka, R.; Yokoi, M.; Shiba, Y. \& Hwang, S. (2007). Development of a groundsource heat pump system with ground heat exchanger utilizing the cast-in-place concrete pile foundations of buildings, ASHRAE Transactions, 2007, 113 (1): pp. 558-566.

Spitler, J. D. (2005). Ground-source heat pump system research - past, present and future. International Journal of HVAC\&R Research, 2005, 11(2): pp. 165-167.

Spitler, J. D.; Cullin, J.; Bernier, M.; Kummert, M.; Cui, P.; Liu, X.; Lee, E. \& Fisher, D. (2009). Preliminary intermodel comparison of ground heat exchanger simulation models. Proceedings of 11th International Conference on Thermal Energy Storage. June 1417. Stockholm, Sweden.

Sutton, M. G.; Nutter, D. W. \& Couvillion, R. J. (2003). A ground resistance for vertical borehole heat exchangers with groundwater flow, J. Energy Resour. ASME, 2003; 125 (3): pp. 183-189.

Xu, X.; Spitler, J. D. (2006). Modeling of Vertical Ground Loop Heat Exchangers with Variable Convective Resistance and Thermal Mass of the Fluid. Proceedings of Ecostock 2006, Pomona, NJ.

Yavuzturk, C. \& Spitler, J. D. (1999). A short time step response factor model for vertical ground loop heat exchangers, ASHRAE Transactions, 1999, 105(2): pp. 475-485.

Yavuzturk, C.; Spitler, J. D.; \& Rees, S. J. (1999). A transient two-dimensional finite volume model for the simulation of vertical U-tube ground heat exchangers, ASHRAE Transactions, 1999, 105 (2): pp. 465-474.

Zeng, H. Y.; Diao, N. R. \& Fang, Z. H. (2002). A finite line-source model for boreholes in geothermal heat exchangers, Heat Transfer-Asian Research, 2002, 31(7): pp. 558567. 
Zeng, H. Y.; Diao, N. R. \& Fang, Z. H. (2003). Heat transfer analysis of boreholes in vertical ground heat exchangers, International Journal of Heat and Mass Transfer, 2003, 46 (23): pp. 4467-4481. 


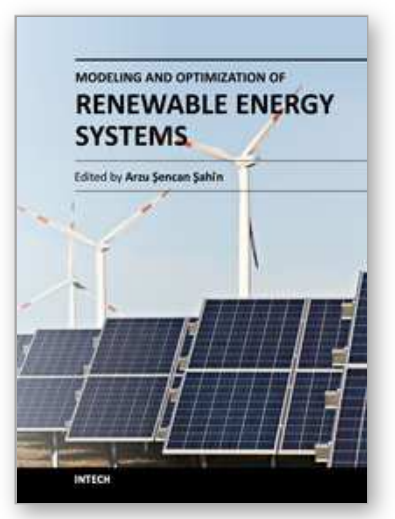

\author{
Modeling and Optimization of Renewable Energy Systems \\ Edited by Dr. Arzu Şencan
}

ISBN 978-953-51-0600-5

Hard cover, 298 pages

Publisher InTech

Published online 11, May, 2012

Published in print edition May, 2012

This book includes solar energy, wind energy, hybrid systems, biofuels, energy management and efficiency, optimization of renewable energy systems and much more. Subsequently, the book presents the physical and technical principles of promising ways of utilizing renewable energies. The authors provide the important data and parameter sets for the major possibilities of renewable energies utilization which allow an economic and environmental assessment. Such an assessment enables us to judge the chances and limits of the multiple options utilizing renewable energy sources. It will provide useful insights in the modeling and optimization of different renewable systems. The primary target audience for the book includes students, researchers, and people working on renewable energy systems.

\title{
How to reference
}

In order to correctly reference this scholarly work, feel free to copy and paste the following:

Yi Man, Ping Cui and Zhaohong Fang (2012). Heat Transfer Modeling of the Ground Heat Exchangers for the Ground-Coupled Heat Pump Systems, Modeling and Optimization of Renewable Energy Systems, Dr. Arzu Şencan (Ed.), ISBN: 978-953-51-0600-5, InTech, Available from: http://www.intechopen.com/books/modelingand-optimization-of-renewable-energy-systems/heat-transfer-modeling-of-the-ground-heat-exchangers-forthe-ground-coupled-heat-pump-system

\section{INTECH}

open science | open minds

\author{
InTech Europe \\ University Campus STeP Ri \\ Slavka Krautzeka 83/A \\ 51000 Rijeka, Croatia \\ Phone: +385 (51) 770447 \\ Fax: +385 (51) 686166 \\ www.intechopen.com
}

\author{
InTech China \\ Unit 405, Office Block, Hotel Equatorial Shanghai \\ No.65, Yan An Road (West), Shanghai, 200040, China \\ 中国上海市延安西路65号上海国际贵都大饭店办公楼405单元 \\ Phone: +86-21-62489820 \\ Fax: +86-21-62489821
}


(C) 2012 The Author(s). Licensee IntechOpen. This is an open access article distributed under the terms of the Creative Commons Attribution 3.0 License, which permits unrestricted use, distribution, and reproduction in any medium, provided the original work is properly cited. 